\title{
Using Direct and Contructive Methods for the Existence of Origami Models with Given Boundary Conditions ${ }^{1}$
}

\author{
R. Geretschläger, S.L. Keeling
}

\begin{abstract}
Whenever a unit square is folded to create an origami model in threedimensional space, the edge of the paper forms a closed curve in space with a total length equal to four units. In this paper, some of the restrictions applicable to such resulting closed curves are derived in the case of classic origami models, in which none of the sections of the folded paper is curved in any way. This allows us to restrict the methods applied to those of classic euclidean geometry. Noting that it is of interest to determine origami models whose edges coincide with a polyline fulfilling the required conditions, we then proceed to show some methods for reconstructing the origami model if the boundary is known. Finally, some concrete reconstructions are demonstrated.
\end{abstract}

Key words and phrases: origami, reconstruction, inverse problem.

\section{Uso de métodos directos y constructivos para la existencia de modelos de origami con condiciones límite dadas}

\section{Resumen}

Siempre que una unidad cuadrada se pliega para crear un modelo de origami en un espacio tridimensional, el borde del papel forma una curva cerrada en el espacio con una longitud total igual a cuatro unidades. En este trabajo, algunas de las restricciones aplicables a estas curvas cerradas resultantes se derivan en el caso de los modelos clásicos de origami, en los que ninguna de las secciones del papel plegado está curvada de ninguna manera. Esto nos permite restringir los métodos aplicados a los de la geometría clásica euclídea. Observando que es de interés determinar modelos de origami cuyos bordes coincidan con una polilínea que cumpla con las condiciones requeridas, procedemos a mostrar algunos métodos para reconstruir el modelo de origami si se conocen los límites. Finalmente, se muestran algunas reconstrucciones concretas.

Palabras y frases clave: origami, reconstrucción, problema inverso.

Recibido 20/05/2019. Aceptado 20/08/2019. 


\section{Introduction}

Whenever a unit square is folded to create an origami model in three-dimensional space, the edge of the paper forms a closed curve in space with a total length equal to four units. It is quite obvious that the model fully determines its edge, i.e. the closed boundary curve of the model. An interesting question resulting in this context is to find out under which circumstances we can turn this around. In other words, when is it possible to derive the structure of the entire model from knowledge of its boundary.

To this purpose, we will restrict ourselves here to classic origami models, in which none of the paper is curved in any way. Restricting consideration to plane sectors on the resulting origami models in this way, it is possible to apply methods of classic euclidean geometry. (Note that this is an alternative to the numerical approach investigated by the authors in [2] for the more general case.) An example of such a reconstruction is shown in Figure 1. The bounding polyline shown on the left yields the simple origami model on the right.

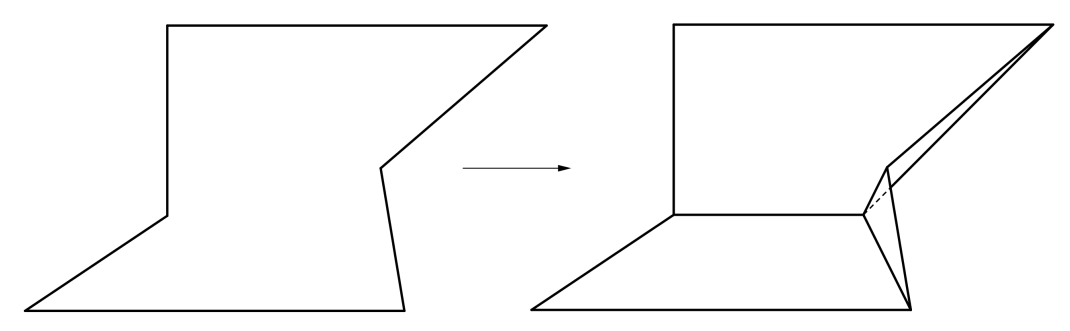

Figure 1: a bounding polyline and its associated origami model

This origami model is certainly not unique, since any sink-fold of the inside peak creates a new origami model with the same bounding polyline. It is, however, reasonable to consider this as a minimal kind of triangulation of the polyline (although two of the four plane sectors are, in fact, quadrilaterals, of course).

We can assume that we will be able to find such triangulations for many other appropriate closed polylines. In fact, it was shown in [1] that this is indeed always the case if some reasonable conditions are met by the boundary of the folded paper, and that a solution to the associated inverse problem can be computed in polynomial time. While such a resulting origami model will generally not be unique, we might naively assume that there should always exist a "simplest" such model in some sense. As we shall see, this will not necessarily be the case.

In this paper, we will attempt to illuminate the question of reconstruction in 
three steps. First of all, we will consider restrictions on the polyline that must be satisfied in order for an origami model with the given polyline as a boundary to exist if we assume that we are starting off from a unit square. Next, we will derive some general basic theorems of reconstruction, which we will then apply in the final section to some concrete reconstructions.

From a mathematical standpoint, we will be using some shortcuts in terminology. A "sheet of paper" will be assumed to be a plane embedded in $\mathbb{R}^{3}$. A "unit square" will be a square section of such a plane with sides of unit length. An "origami model" will be a connected collection of plane objects that can result from the unit square by folding procedures. (What exactly constitutes a legitimate folding procedure will not be precisely defined here, but the meaning in the given context is assumed to be clear.) The plane sections of the origami model will be referred to as "facets" of the origami model, and its edges will be referred to as "creases" if they are not part of the bounding polyline.

For the moment, we will assume that the origami models are in general not flat, but rather of a general three-dimensional nature. The special case of flat-folding will be touched upon in section 5 .

Before we begin, it may be of interest to note that we are using the unit square as a starting-off point here because of its traditional nature in practical origami. An interesting alternative would be to consider what happens with a unit circle as the folding medium, and what happens to its boundary. It is quite obvious that the restrictions on the circle will be quite severe. For now, however, practical restrictions in space and time limit us to one type of folding medium, and the discussion of folding the unit circle will have to wait for some other time and place.

\section{Restrictions on the Polyline}

There are numerous restrictions both to polylines that could possibly be boundaries of origami models folded from a unit square, and to the origami models corresponding to such polylines. Some of these restrictions are quite obvious. For instance, not only must the total length of the polyline be equal to four units, the fact that the edges of the unit square are at right angles to each other in the corners must obviously yield restrictions on the line segments of the polylines that result from these edges. The polyline must therefore consist of four sections, each of unit length, obeying some specific corner angle restrictions. Furthermore, the area of the unit square is equal to one square unit, and this must obviously also be the case for the sum of the areas of the facets of the origami model. In this section, we will consider some of the restrictions on the polyline that make it a candidate for the boundary of an origami 
model.

First of all, we require the following, seemingly obvious, preliminary result.

Theorem 1: Let $P$ and $Q$ be points on a sheet of paper s, and let $P^{\prime}$ and $Q^{\prime}$ be the points in which they come to lie in some origami model folded from a unit square embedded in $s$ and containing $P$ and $Q$. Then $P^{\prime} Q^{\prime} \leq P Q$ certainly holds.

Proof: Consider the line segment joining $P$ to $Q$ in $s$. If we let $\left\{c_{i}\right\}$ denote the set of all lines $c_{i}$ in $s$, sections of which map to creases $c_{i}^{\prime}$ in the origami model, we note that $P Q$ intersects some of these lines $c_{i}$. Assume that $P Q$ intersects $c_{1}, c_{2}, \ldots, c_{k}$ in points $C_{1}, C_{2}, \ldots, C_{k}$, with indices chosen such that the points $P, C_{1}, C_{2}, \ldots, C_{k}, Q$ lie on $P Q$ in this order. If $C_{1}^{\prime}, C_{2}^{\prime}, \ldots, C_{k}^{\prime}$ are their maps in the origami model, we have a polyline $P^{\prime} C_{1}^{\prime}, C_{1}^{\prime} C_{2}^{\prime}, \ldots, C_{k}^{\prime} Q^{\prime}$ joining $P^{\prime}$ and $Q^{\prime}$ in the model in such a way that each segment of the polyline lies completely in a facet of the origami model, as illustrated in Figure 2.

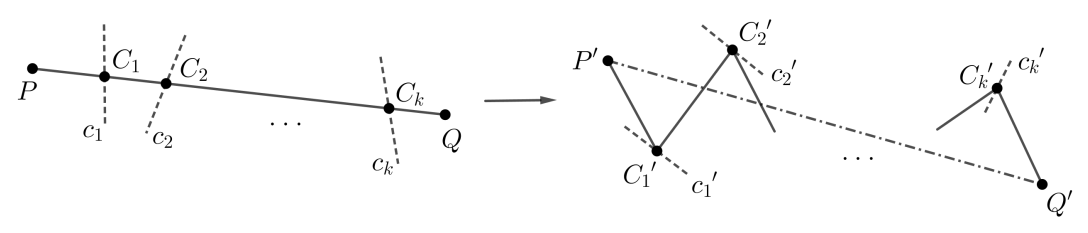

Figure 2: folding $P Q$

This means that the length of each segment of the polyline is equal to the length of the respective segment in $s$, and the total length of the polyline is therefore equal to the length of $P Q$. By the triangle inequality, the distance $P^{\prime} Q^{\prime}$ in $\mathbb{R}^{3}$ is certainly not greater than the length of the polyline joining $P^{\prime}$ to $Q^{\prime}$ (in fact, it is less if the set $\left\{c_{i}\right\}$ is not empty), and the proof is complete.

A related result is the following.

Theorem 2: Let $r_{1}$ and $r_{2}$ be line segments on a unit square $u$ with a common endpoint $E$, and let $\varphi$ be the angle contained by the two line segments (with $0^{\circ}<$ $\left.\varphi \leq 180^{\circ}\right)$. Let $E^{\prime}$ be the point in which $E$ comes to lie in some origami model folded from $u$, and let $r_{1}^{\prime}$ and $r_{2}^{\prime}$ be the line segments emanating from $E^{\prime}$ resulting from the parts of $r_{1}$ and $r_{2}$ next to $E$ that come to lie in a common facet with $E^{\prime}$. (Note that 
$r_{1}^{\prime}$ and $r_{2}^{\prime}$ need not lie in a common facet.) Let $\varphi^{\prime}$ be the angle contained by $r_{1}^{\prime}$ and $r_{2}^{\prime}$ (with $\left.0^{\circ} \leq \varphi^{\prime} \leq 180^{\circ}\right)$. Then $\varphi^{\prime} \leq \varphi$ certainly holds.

Proof: Since $r_{1}^{\prime}$ and $r_{2}^{\prime}$ each lie completely in a facet of the origami model with $E$, there exist points $X_{1}^{\prime}$ and $X_{2}^{\prime}$ on $r_{1}^{\prime}$ and $r_{2}^{\prime}$ respectively, equidistant from $E$, as shown in Figure 3. Letting $X_{1}$ and $X_{2}$ denote their corresponding points on $r_{1}$ and $r_{2}$ respectively, it follows that $E X_{1}=E X_{2}=E^{\prime} X_{1}^{\prime}=E^{\prime} X_{2}^{\prime}$ holds. Note that $\varphi^{\prime}=\varphi$ will always hold if there is no crease through $E$ in the origami model, i.e. if $r_{1}^{\prime}$ and $r_{2}^{\prime}$ lie in the same facet. If there is, we will certainly have $\varphi^{\prime}<\varphi$.

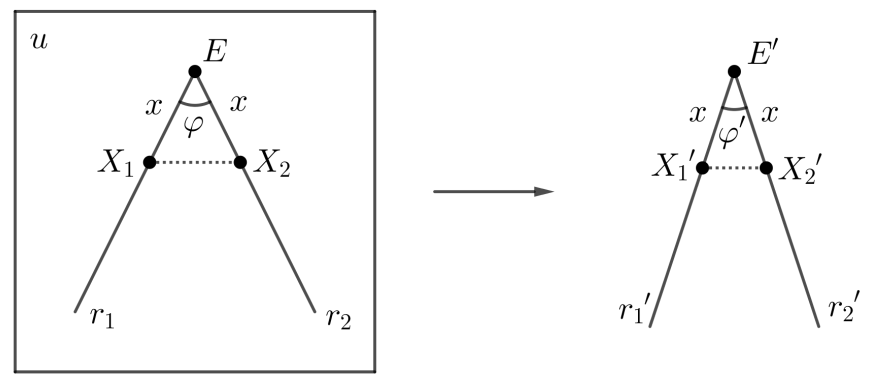

Figure 3: folding an angle

Naming this distance $x$ and applying Theorem 2, we obtain

$$
\cos \varphi^{\prime}=\frac{2 x^{2}-X_{1}^{\prime} X_{2}^{\prime 2}}{2 x^{2}} \geq \frac{2 x^{2}-X_{1} X_{2}^{2}}{2 x^{2}}=\cos \varphi
$$

from triangles $E X_{1} X_{2}$ and $E^{\prime} X_{1}^{\prime} X_{2}^{\prime}$, which yields $\varphi^{\prime} \leq \varphi$, as claimed.

With these two preliminary observations at hand, we are now ready to characterize the boundaries of origami models folded from unit squares.

If we are given a unit square $u$ with corners $A_{1}, A_{2}, A_{3}$ and $A_{4}$, and $u$ is folded into an origami model with the boundary $b$ as illustrated in Figure 4, the boundary $b$ is certainly a closed polyline with the total length four, on which points $A_{i}^{\prime}$ corresponding to the respective corners of $u$ must lie. Since the sides of $u$ meet at right angles in each corner of $u$, and the angles in an origami model must be smaller than (or equal to) the corresponding angles in the original unit square $u$ according to Theorem 2, we see that the angles at which segments of the polyline boundary meet in the points $A_{i}^{\prime}$ must be right or acute. We can therefore sum up these observations in the first boundary condition that $b$ must fulfill. 
(B1) The boundary $b$ of an origami model is a closed polyline of length four. There exist four vertices $A_{1}^{\prime}, A_{2}^{\prime}, A_{3}^{\prime}$ and $A_{4}^{\prime}$ on the polyline such that the sum of the lengths of the line segments joining each pair $A_{i}^{\prime}$ and $A_{i+1}^{\prime}$ (with all indices taken modulo 4) equals one and the angles in which the segments of $b$ meet in each of the vertices $A_{i}^{\prime}$ are either right or acute.

As a next step, we consider points $X$ and $Y$ on the sides $A_{1} A_{2}$ and $A_{2} A_{3}$ of $u$ respectively, as in Figure 4.

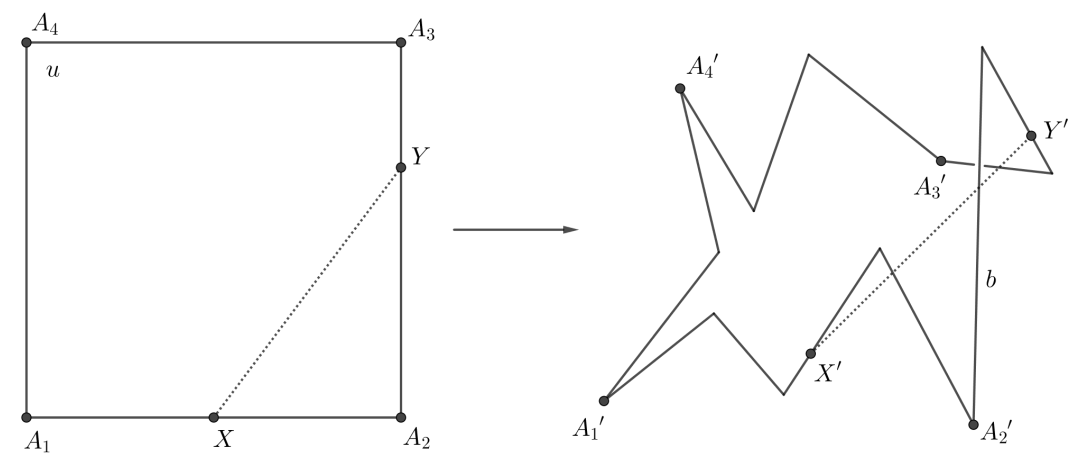

Figure 4:

The length of the line segment is given by the expression

$$
X Y=\sqrt{A_{2} X^{2}+A_{2} Y^{2}} .
$$

By Theorem 2, we know that $X^{\prime} Y^{\prime} \leq X Y$ certainly holds, and since the length of segment $A_{2} X$ is equal to the sum of the lengths of the segments joining $A_{2}^{\prime}$ and $X^{\prime}$ (as the length of $A_{2} Y$ is equal to the sum of the lengths of the segments joining $A_{2}^{\prime}$ and $\left.Y^{\prime}\right), b$ must also fulfill the following second boundary condition.

(B2) If $X^{\prime}$ is a point between $A_{i-1}^{\prime}$ and $A_{i}^{\prime}$ on $b$ and $Y^{\prime}$ a point between $A_{i}^{\prime}$ and $A_{i+1}^{\prime}$ (indices taken modulo 4), and $x$ the sum of the lengths of the line segments joining $X^{\prime}$ and $A_{i}^{\prime}$ and $y$ the sum of the lengths of the line segments joining $Y^{\prime}$ and $A_{i}^{\prime}$, the distance between $X^{\prime}$ and $Y^{\prime}$ must fulfill the condition $X^{\prime} Y^{\prime} \leq \sqrt{x^{2}+y^{2}}$.

A similar condition must also follow for points on opposite sides of $u$, which we consider in Figure 5.

Let $X$ and $Y$ be points on the sides $A_{1} A_{2}$ and $A_{3} A_{4}$ of $u$ respectively. In this 


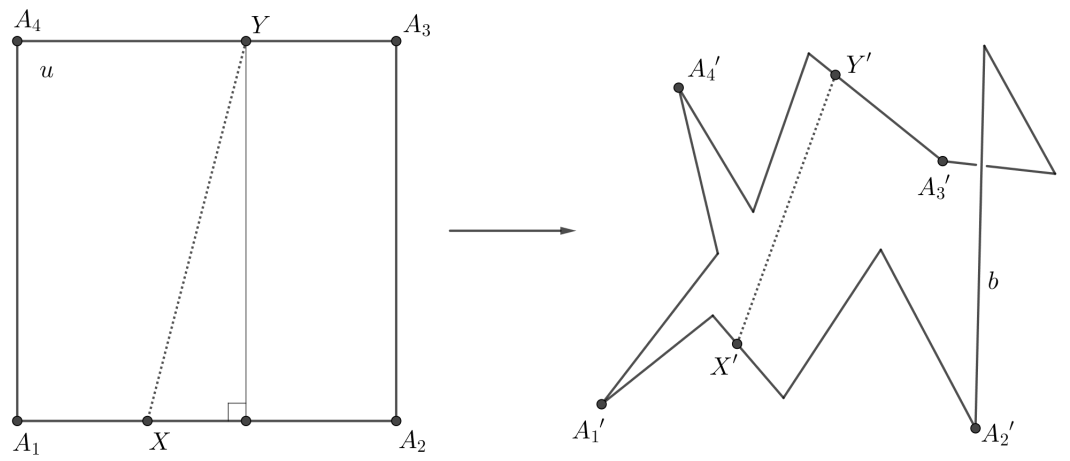

Figure 5: $X Y$ on a folded square

case, the length of $X Y$ is given by the expression

$$
X Y=\sqrt{1^{2}+\left|A_{1} X-A_{4} Y\right|^{2}} .
$$

The analogous argument to the one just given for the second boundary condition then yields the third boundary condition.

(B3) If $X^{\prime}$ is a point between $A_{i}^{\prime}$ and $A_{i+1}^{\prime}$ on $b$ and $Y^{\prime}$ a point between $A_{i-1}^{\prime}$ and $A_{i-2}^{\prime}$ (indices taken modulo 4), and $x$ the sum of the lengths of the line segments joining $A_{i}^{\prime}$ and $X^{\prime}$ and $y$ the sum of the lengths of the line segments joining $A_{i-1}^{\prime}$ and $Y^{\prime}$, the distance between $X^{\prime}$ and $Y^{\prime}$ must fulfill the condition $X^{\prime} Y^{\prime} \leq \sqrt{1+|x-y|^{2}}$.

\section{Theorems of Reconstruction}

If the bounding polyline of an origami model is given, the creases of the model must obey certain laws with respect to the line segments comprising the polyline. In this section, we will concern ourselves with the identification of possible positions of such creases relative to the segments of the polyline. As a first step toward a practical realisation of a reconstruction, we must consider how the edge of the unit square can be folded and how the corners of the unit square can be folded.

Theorem 3: Let $s$ be a sheet of paper and $\ell$ a line in $s$. The sheet $s$ is folded by a single fold on a crease $c$ at a dihedral angle $\theta\left(0^{\circ} \leq \theta<180^{\circ}\right)$ in such a way that $c$ intersects $\ell$ in a point $V$ at an acute angle $\varphi\left(0^{\circ}<\varphi \leq 90^{\circ}\right)$. Assume that $c$ remains fixed in $\mathbb{R}^{3}$ and the two parts of $\ell$ are mapped to rays $\ell_{1}$ and $\ell_{2}$ by the fold. Then $c$ is perpendicular to the angle bisector of $\ell_{1}$ and $\ell_{2}$. 
Proof: Let us assume that $s$ lies in the $[x y]$ coordinate plane, with $c$ in the $x$-axis and $V$ in the origin, as shown in Figure 6. Line $\ell$ is composed of points with coordinates $(x, y, 0)$ satisfying the equation $x=a y$ for some real parameter $a=\cot \varphi$. Specifically, points $E_{1}(a, 1,0)$ and $E_{2}(-a,-1,0)$ lie on $\ell$. Folding $s$ at the dihedral angle $\theta$, we place the folded sheet $s^{\prime}$ in such a way that $c$ remains on the $x$-axis and $s^{\prime}$ is symmetric with respect to the $[x z]$ coordinate plane. $E_{1}$ then folds (i.e. maps) to $E_{1}^{\prime}\left(\cot \varphi, \sin \frac{\theta}{2},-\cos \frac{\theta}{2}\right)$ and $E_{2}$ to $E_{2}^{\prime}\left(-\cot \varphi,-\sin \frac{\theta}{2},-\cos \frac{\theta}{2}\right)$.

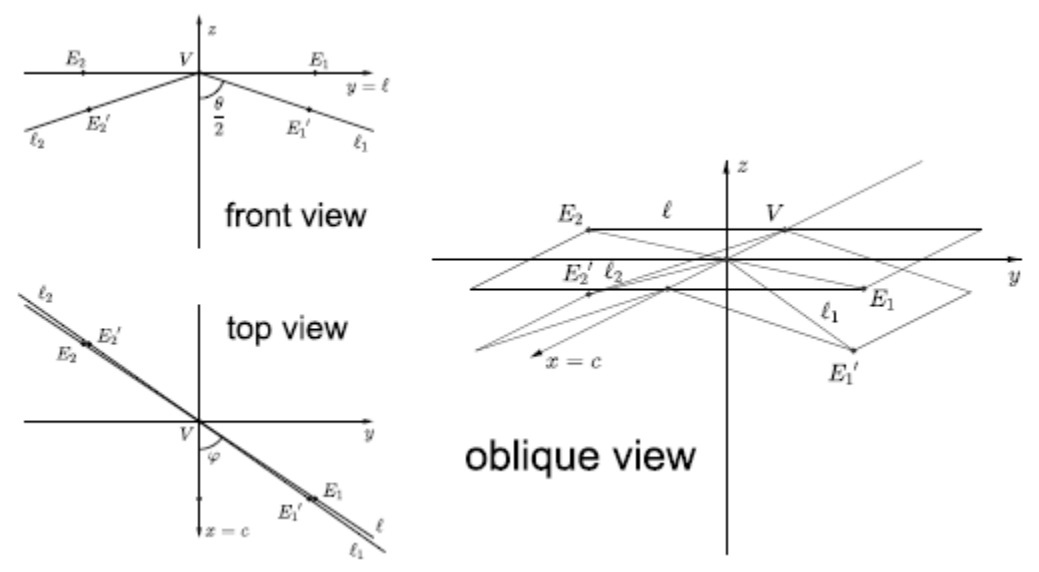

Figure 6: first theorem of reconstruction

The ray $\ell_{1}=\overrightarrow{V E_{1}^{\prime}}$ is therefore symmetric to $\ell_{2}=\overrightarrow{V E_{2}^{\prime}}$ with respect to the $z$ axis. Since the angle bisector of $\ell_{1}$ and $\ell_{2}$ therefore lies in the $z$-axis, it is certainly perpendicular to the crease $c$, which lies in the $x$-axis, as claimed.

An immediate consequence of this result is the following first step towards actual reconstruction of origami models from given boundaries.

Theorem 4: If an edge e of a unit square is folded by a single fold such that a point $E$ divides e into two segments $e_{1}$ and $e_{2}$ that map to $e_{1}^{\prime}$ and $e_{2}^{\prime}$ respectively, and the angle contained by $e_{1}^{\prime}$ and $e_{2}^{\prime}$ is equal to $\varepsilon$ with $0^{\circ}<\varepsilon<180^{\circ}$, the resulting crease $c$ lies on one of the lines through $E$ contained in the plane perpendicular to the angle bisector of $e_{1}^{\prime}$ and $e_{2}^{\prime}$.

Knowing this, we must now turn our attention to the slightly more difficult case of folding the corner. 
Theorem 5: If a corner of a unit square is folded by a single fold such that the edges $e_{1}$ and $e_{2}$ meeting in the corner $A$ are mapped to segments $e_{1}^{\prime}$ and $e_{2}^{\prime}$ respectively, meeting in $A^{\prime}$, the resulting crease $c$ is a generating line of a second-degree cone $\Gamma$. The axis $a_{\Gamma}$ of $\Gamma$ is the angle bisector of the angle contained by $e_{1}^{\prime}$ and $e_{2}^{\prime}$.

Proof: In order to see this, we place the point $A^{\prime}$ and the segments $e_{1}^{\prime}$ and $e_{2}^{\prime}$ in a coordinate system as shown in Figure 7.

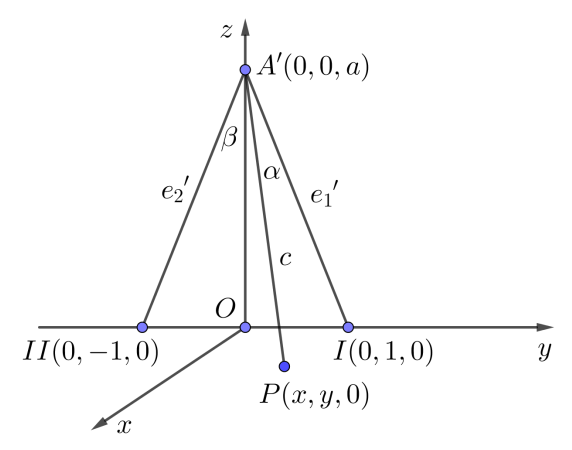

Figure 7: second theorem of reconstruction

The point $A^{\prime}$ is placed on the $z$-axis with coordinates $A^{\prime}(0,0, a)$ while $e_{1}^{\prime}$ and $e_{2}^{\prime}$ are placed in the $y z$-plane in such a way that $e_{1}^{\prime}$ intersects the $y$-axis in the point $I(0,1,0)$ and $e_{2}^{\prime}$ intersects the $y$-axis in the point $I I(0,-1,0)$. A crease $c$ emanating from $A^{\prime}$ intersects the $x y$-plane in a point $P$ with coordinates $(x, y, 0)$, and if we denote the angles that $c$ contains with $e_{1}^{\prime}$ and $e_{2}^{\prime}$ as $\angle c e_{1}=\alpha$ and $\angle c e_{2}^{\prime}=\beta$, we have $\alpha+\beta=90^{\circ}$.

Standard calculation yields

$$
\cos \alpha=\frac{a^{2}+y}{\sqrt{x^{2}+y^{2}+a^{2}} \cdot \sqrt{a^{2}+1}} \quad \text { and } \quad \cos \beta=\frac{a^{2}-y}{\sqrt{x^{2}+y^{2}+a^{2}} \cdot \sqrt{a^{2}+1}},
$$

and since $\cos (\alpha+\beta)=0$ must hold, we obtain $\cos \alpha \cos \beta-\sin \alpha \sin \beta=0$. Substituting the values of $\cos \alpha$ and $\cos \beta$ in this equation, we obtain an expression that simplifies to

$$
a^{4}+y^{2}=a^{2}\left(x^{2}+y^{2}+1\right)+x^{2} .
$$

Since the $x y$-plane intersects the cone in a quadratic curve (an ellipse, in fact), the cone itself is also quadratic, as claimed. 
We name this cone the vertex cone in $A$ and $e_{1}^{\prime}$ and $e_{2}^{\prime}$ the focus lines of the vertex cone. Note that the points in which the focus lines intersect the $x y$-plane are not the foci of the ellipse in the $x y$-plane, but the role of these lines is analogous to the role of the foci of an ellipse, since the sum of the angles $\angle c e_{1}=\alpha^{\prime}$ and $\angle c e_{2}^{\prime}=\beta$ is constant.

\section{Reconstructing a Model from its Boundary}

In this section, we turn our attention to concrete reconstructions of some simple origami models. As a first example, we will now revisit the origami model and boundary from Figure 1. As we see in Figure 8, the bounding polyline $b$ is a hexagon with six vertices. As shown, we place the polyline in a system of coordinates in such a way that the coordinates of the vertices of $b$ are $A_{1}\left(0,0, \frac{1}{2}\right), A_{2}\left(0,1, \frac{1}{2}\right), I\left(\frac{1}{3}, \frac{2}{3}, \frac{1}{3}\right)$, $A_{3}\left(\frac{1}{2}, 1,0\right), A_{4}\left(\frac{1}{2}, 0,0\right)$ and $I I=O(0,0,0)$.

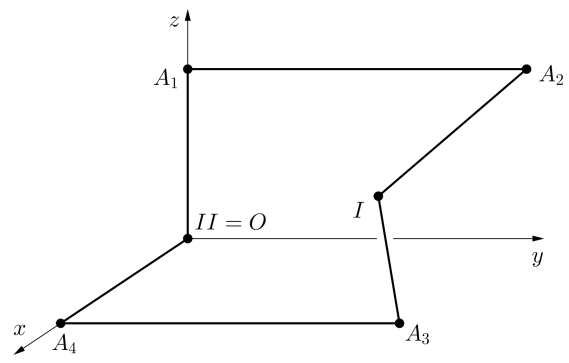

Figure 8: bounding polyline in a system of coordinates

Note that $A_{1} I I=A_{4} I I=A_{2} I=A_{3} I=\frac{1}{2}$ and $A_{1} A_{2}=A_{3} A_{4}=1$ certainly holds for these specific coordinates. The total length of $b$ is therefore equal to four, as required.

As a first step in reconstructing an origami model from this polyline, we must identify which four of the six vertices of the polyline are candidates for the corners of the folding square. In this particular case, it is quite obvious, since we have $A_{1} A_{2}=A_{3} A_{4}=1$, and $A_{1}, A_{2}, A_{3}$ and $A_{4}$ must therefore be the corners of the square.

Next, we note that the angles between successive segments of $b$ in $A_{1}$ and $A_{4}$ are right. Since there are no folds in the boundary between $A_{1}$ and $A_{2}$ or between $A_{1}$ and $I I$, the triangle $A_{1} A_{2} I I$ cannot contain a crease, and the analogous result also follows for triangle $A_{4} A_{3} I I$. A possible candidate for a crease of the reconstructed model emanating from $I I$ (under the simplifying assumption that only one crease of 
the model ends in $I I$ ) is therefore the line in which the planes of these two triangles intersect, and since $A_{1} A_{2} I I$ lies in the $y z$-plane and $A_{4} A_{3} I I$ lies in the $x y$-plane, this line of intersection is the $y$-axis. This is illustrated in Figure 9.

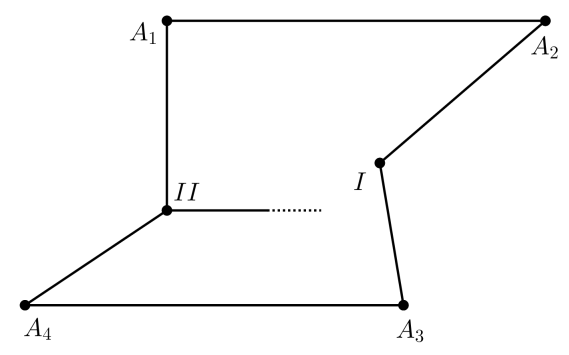

Figure 9: first forced crease

We still require creases emanating from $A_{2}, A_{3}$ and $I$. Since $I$ is not a corner of the folding square, a crease emanating from $I$ must lie in the plane perpendicular to the angle bisector of $\angle A_{2} I A_{3}$ by Theorem 3 . Since $I A_{2}=I A_{3}=\frac{1}{2}$, this angle bisector joins $I$ with the mid-point $\left(\frac{1}{4}, 1, \frac{1}{4}\right)$ of $A_{2} A_{3}$, and the equation of this plane is therefore $x-4 y+z=-2$. If the crease emanating from $I$ is to terminate in a common end-point with the crease emanating from $I I$, this common point $I I I$ must be the point in which the $y$-axis intersects this plane, i.e. the point $\operatorname{III}\left(0, \frac{1}{2}, 0\right)$, as shown in Figure 10.

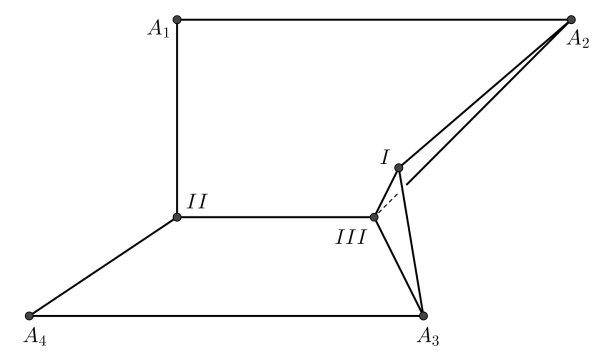

Figure 10: possible reconstruction

We can now check to make sure that this internal point $I I I$ does indeed complete a legitimate origami model. The angles $\angle A_{1} A_{2} I I I$ and $\angle A_{4} A_{3} I I I$ are each equal to $45^{\circ}$, and it is easy to calculate that the same is true of $\angle I A_{2} I I I$ and $\angle I A_{3} I I I$. Triangles $I A_{2} I I I$ and $I A_{3} I I I$ are therefore both isosceles right triangles with sides of length $\frac{1}{2}$. Quadrilaterals $A_{1} A_{2} I I I I I$ and $A_{4} A_{3} I I I I I$ are congruent right angled trapezoids with sides of length $1, \frac{\sqrt{2}}{2}, \frac{1}{2}$ and $\frac{1}{2}$ respectively, and these shapes can 
be unfolded to yield the unit square. We see that we have indeed reconstructed a possible origami model with the given boundary $b$.

By Theorem 3, we note that the crease $A_{2} I I I$ is a generating line of the vertex cone in $A_{2}$ with focus lines $A_{2} A_{1}$ and $A_{2} I$, as is the crease $A_{3} I I I$ of the vertex cone in $A_{3}$ with focus lines $A_{3} A_{4}$ and $A_{3} I$.

As a next example, we consider a four-pointed symmetrical star as a bounding polyline $b$. As shown in Figure 11, the polyline is composed of eight line segments of equal length, and we can assume that this length equals $\frac{1}{2}$, since the perimeter of the star then equals four, as required. The star has four axes of symmetry, and in order for vertices to exist, in which the line segments contain angles less than $90^{\circ}$ as required, the star cannot be convex.

As shown in the right-hand part of Figure 11, we can place the star into a system of coordinates such that the vertices of $b$ are $A_{1}(a, 0,0), I(b, b, 0), A_{2}(0, a, 0)$, $I I(-b, b, 0), A_{3}(-a, 0,0), I I I(-b,-b, 0), A_{4}(0,-a, 0)$ and $I V(b,-b, 0)$ with $a_{i}<\frac{\sqrt{2}}{2}$ (since $O A_{1} A_{2}$ is an isosceles right triangle with a hypotenuse shorter than $A_{1} I+I A_{2}=$ 1 ), $b<\frac{a}{2}$ (since the star is certainly not convex) and

$$
\frac{1}{2}=A_{1} I=\sqrt{(a-b)^{2}+b^{2}} \quad \Longleftrightarrow \quad a=b+\sqrt{\frac{1}{4}-b^{2}}
$$

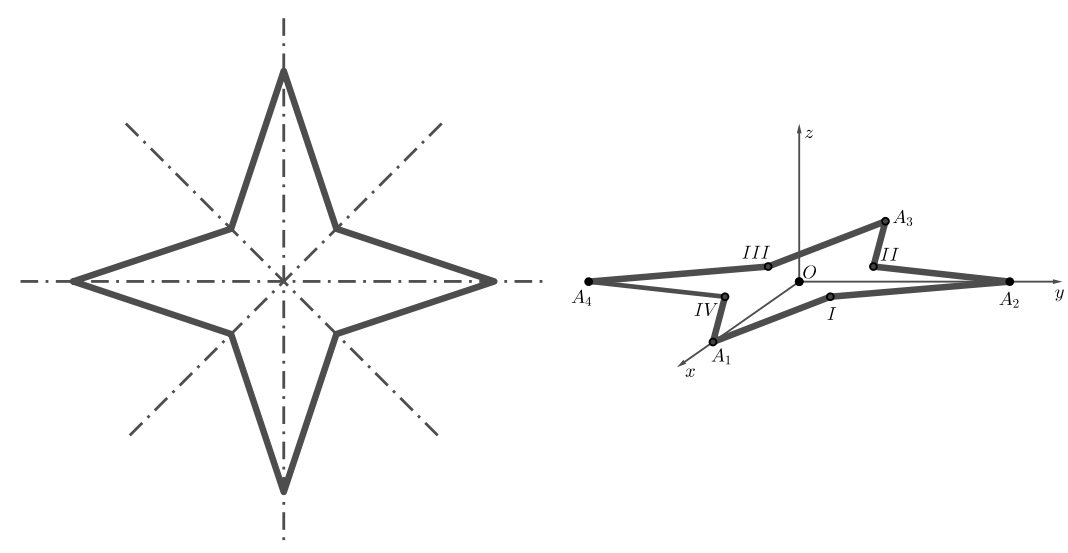

Figure 11: boundary star in the $x y$ plane

It will prove useful to refer to the acute angles in this star as $2 \varphi$, which means that the obtuse (external) angles equal $90^{\circ}+2 \varphi$. 
$A_{1}, A_{2}, A_{3}$ and $A_{4}$ must be the vertices in which the corners of the folding square will lie, since the angles contained by the line segments in these points are acute, whereas the angles contained in $I, I I, I I I$ and $I V$ are obtuse.

There are now several ways in which we could choose to proceed. On one hand, the fact that $b$ is a plane figure seems to imply that there could be a flat-folding origami model with this boundary. For the moment, however, we will put this idea on hold, in favor of another quite elementary observation.

Noting that the star has four axes of symmetry, it seems reasonable to hope that we can find an origami model with this star as a boundary $b$ with four planes of symmetry, namely the four planes through the axes of symmetry of the star, perpendicular to the plane of the star. These are the $x z$ and $y z$ coordinate planes and the planes joining the $z$-axis with points $I$ and $I I$ respectively. By Theorem 3, we know that a crease ending in $I$ must lie in a plane perpendicular to the angle bisector of $\angle A_{1} I A_{2}$ (if there is only a single crease through this point, which we assume for the sake of simplicity), and because of the vertical plane of symmetry passing through this point, the crease must also be vertical, i.e. parallel to the $z$ axis, or in other words, perpendicular to the plane of the star $b$. Since the same will hold true for the creases ending in $I I, I I I$ and $I V$ under the analogous simplifying assumptions, the creases must lie as suggested in Figure 12.

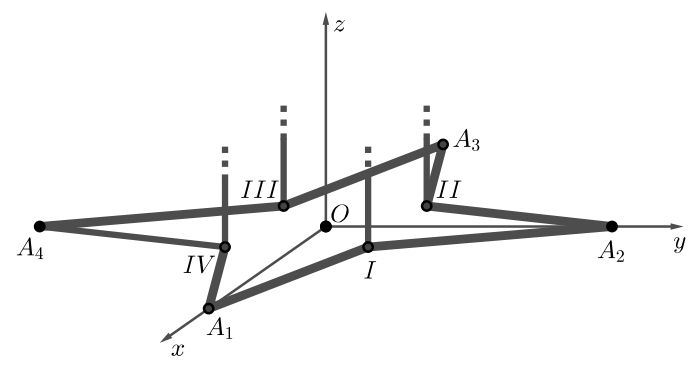

Figure 12: possible creases perpendicular to the $x y$ plane

As a next step, we can now consider which crease(s) might end in $A_{1}$ (and therefore, by the assumed symmetry, in $A_{2}, A_{3}$ and $A_{4}$ ). If there is only one crease ending in $A_{1}$, this must be perpendicular to the plane of $b$, as this is the case for the two facets of the model with edges $A_{1} I$ and $A_{1} I V$. Since such a crease would be perpendicular both to $A_{1} I$ and $A_{1} I V$, unfolding such a crease would yield collinear points $I, A_{1}$ and $I V$, in contradiction to the fact that $A_{1}$ must unfold to a corner of the unit square. There must therefore be more than one crease ending in $A_{1}$.

Since the model is assumed to be symmetrical with respect to the $x z$ coordinate 
plane, such folds must themselves be symmetrical with respect to the $x z$ plane, and it seems that a model as shown in Figure 13 should result.

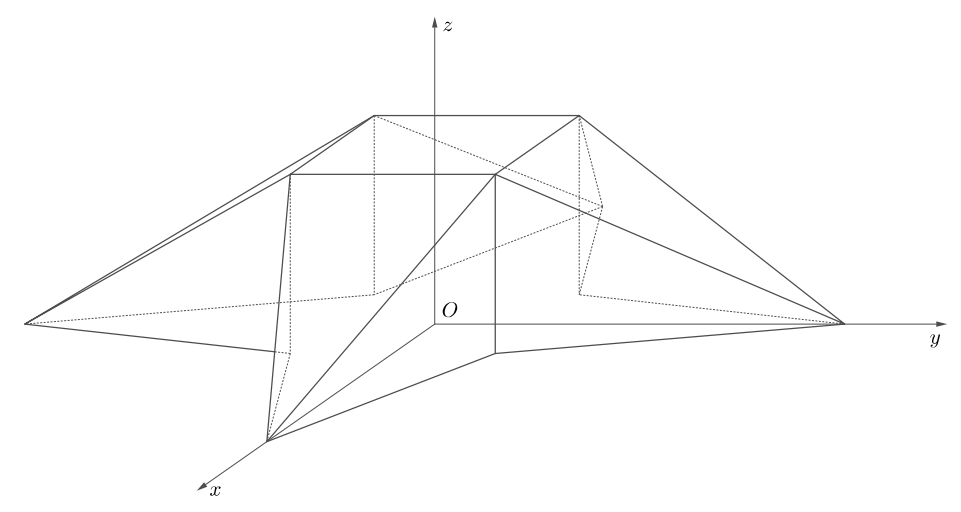

Figure 13: possible solid reconstruction from star boundary

It remains to be shown that such a model is actually foldable from the unit square. In order to see this, we consider the angles in the vertex $A_{1}$, as shown in Figure 14. As in this figure, we let $P$ denote the upper terminus of the vertical crease ending in $I, Q$ the orthogonal projection of $P$ on the $x z$ coordinate plane, and $R$ the orthogonal projection of $I$ on the $x$-axis. Furthermore, having already named $\angle R A_{1} I=\varphi$, we can now also name the angle $\angle P A_{1} I=\alpha$ and the line segment $P A_{1}=p$.

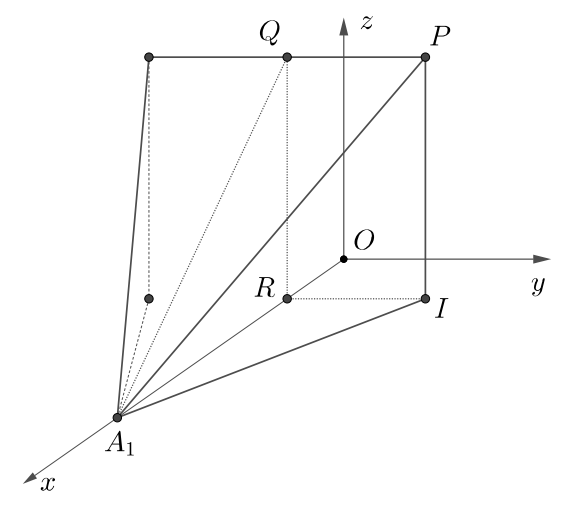

Figure 14: front point of the reconstruction

Since $A_{1}$ unfolds to a corner of the unit square and the model is symmetric with respect to the $x z$ plane, we must have $\angle P A_{1} Q=45^{\circ}-\alpha$. Having established these 
designations, we have

$$
\sin \varphi=\frac{I R}{I A_{1}}=\frac{P Q}{I A_{1}}=\frac{p \cdot \sin \left(45^{\circ}-\alpha\right)}{p \cdot \cos \alpha},
$$

and some easy calculation shows that this is equivalent to

$$
\tan \alpha=1-\sqrt{2} \cdot \sin \varphi .
$$

Since both $\alpha$ and $\varphi$ lie between $0^{\circ}$ and $45^{\circ}$, every possible angle $\varphi$ therefore uniquely determines a corresponding value of $\alpha$ (and vice versa), and a possible origami model therefore certainly exists, since the model will be completed by a square in a plane perpendicular to the $z$-axis, as shown in Figure 13 because of the resulting symmetry.

\section{Reconstructing Flat-folding Models}

Obviously, any flat origami model has a plane boundary, and we therefore have a special case given if we are confronted with a boundary $b$ which lies completely in a plane. In such a case, it seems obvious to consider the possibility of a flat origami model as a candidate for the solution of the reconstruction problem.

Of course, any flat-foldable crease pattern must obey the criteria of Maekawa's Theorem and Kawasaki's theorem, i.e. the number of mountain folds and valley folds in each vertex must differ by two and the sums of alternating angles in each vertex must equal $180^{\circ}$. On the one hand, these are fairly strict constraints for any reconstruction problem, but on the other hand, this very fact makes them very useful tools in finding a concrete solution to such a problem.

As was already mentioned earlier, a four-pointed star with acute angles $2 \varphi$ is an interesting candidate as the boundary $b$ for this type of reconstruction. If we wish to keep the number of folds small, it seems reasonable to hope that we can find a model in which only one crease ends in each of the eight vertices of $b$. If the acute angle is to equal $2 \varphi$, and the fold is to be flat, this means that we must fold one edge of the unit square by an angle of $\frac{1}{2} \cdot\left(90^{\circ}-2 \varphi\right)=45^{\circ}-\varphi$. This is illustrated in Figure 15 .

Furthermore, since the obtuse angles must emanate from the points corresponding to the mid-points of the sides of the unit square, knowing that the obtuse angles must be equal to $90^{\circ}+2 \varphi$, we must fold one half of the edge of the unit square by an angle of $\frac{1}{2} \cdot\left(180^{\circ}-\left(90^{\circ}+2 \varphi\right)\right)=45^{\circ}-\varphi$ as is also illustrated in Figure 15. Since these two angles are equal, the resulting creases will be parallel.

Because of the rotational symmetry of the unit square, this gives us a total of eight creases, of which each one emanating from the mid-point of an edge of the unit 


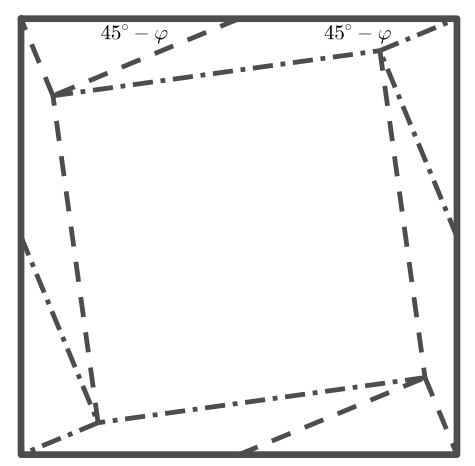

Figure 15: crease pattern for flat model from star boundary

square intersects the adjoining one emanating from the corner. The four resulting points of intersection must therefore be vertices of the folding pattern, and since there must be an even number of creases emanating from each such vertex, it seems obvious to consider the crease pattern completed by the sides of this square. Applying symmetry and Maekawa's Theorem gives us the crease pattern shown in Figure 15. (Or else, of course, we can also get the pattern resulting from the exchange of all mountain folds for valley folds and vice versa.) Noting that the angles of the square equal $90^{\circ}$, as do the angles of the small triangles in the corners of the interior square, we see that the angle constraints of Kawasaki's theorem are also fulfilled, and the crease pattern will indeed fold flat. The result is shown in Figure 16.
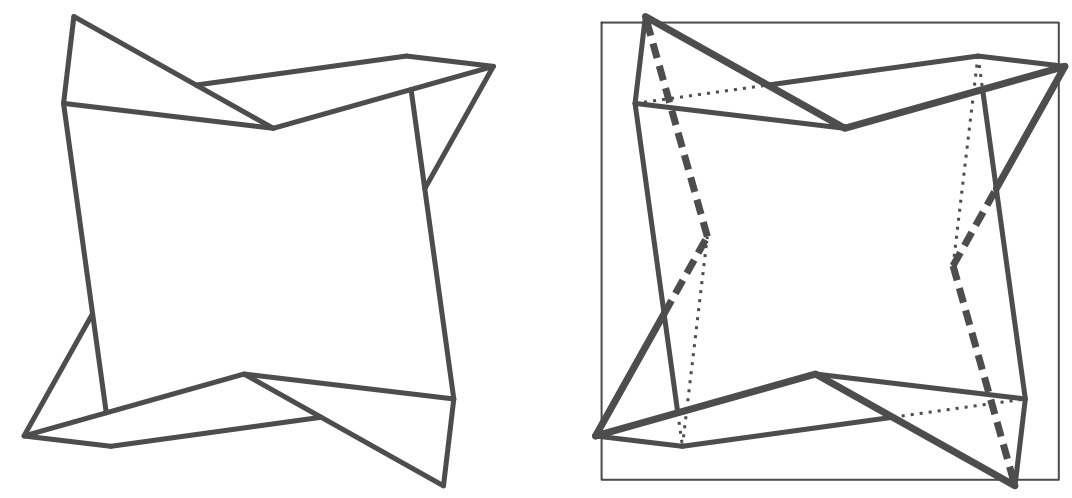

Figure 16: flat reconstruction from star boundary

In the left-hand figure, we see only the visible parts of the creases and the edge 
of the paper. In the right-hand figure, the lines not visible here are also added, with the sections belonging to the border $b$ drawn in thick dashed lines and the invisible crease parts drawn as thin dotted lines. Also, the relative position of the original unit square is shown by thin full lines.

This brings us to an interesting point. While we may have expected some kind of uniqueness when we began looking for solutions to the boundary problem, we have discovered a counter-example in the four-pointed star. The two models that result by assuming the existence of a certain type of symmetry on the one hand, and a flatfolding model on the other, are obviously quite different, but both as simple (in the sense of being somehow irreducible) as can reasonably be expected. This suggests a number of interesting topics for further study.

- Is there always a flat-folding model with the border $b$ fulfilling the restrictions stated there, if $b$ lies completely in a plane? Is there always a non-flat-folding model of this type, if $b$ lies completely in a plane? (Note that this does not necessarily follow from the existence of a flat-folding model, since a sink fold of a flat-folding model is again flat-folding, and sink folds are the only obvious ways to create additional solutions to the boundary problem from given known solutions.)

- Is some kind of "uniqueness" of the solution of the boundary problem given in the case of boundaries that do not lie completely in a plane? How would we define such a type of "uniqueness"?

\section{Conclusion and Topics for Further Study}

It is clear that we have only scratched the surface of this topic so far. The issue of reconstructing origami models from their boundaries promises to be quite fruitful, and many interesting results seem to lie ahead. Some other questions we could ask in this context include the following.

- What numerical properties of the "simplest" model with a given boundary can be derived from the properties of the boundary. In other words, what can we say about the numbers of vertices, creases and facets of such a model if we know the properties of the boundary? Do the smallest such values necessarily correlate with the "simplest" origami model with a certain given boundary?

- What restrictions on the model can be derived from known properties of the boundary? For instance, can we derive any interesting inferences about the 
radius of the smallest sphere surrounding an origami model from the measurements of the boundary, without first actually finding the model itself?

- Is there some way to systematize the reconstruction of an origami model from the boundary by applying the theorems of reconstruction in a systematic manner? Could we develop a program to automatically derive an origami model with a given boundary? It is known that the problem is solvable in polynomial time, but what would a program look like that actually creates the model?

Finally, recall that it is also of interest to consider how we might solve the problem of reconstruction from the boundary with methods other than those derived from classic euclidean ideas. As was already mentioned, a possible numerical approach to this question is suggested in [2].

\section{References}

[1] Demaine E.D. and Ku J.S., Filling a hole in a crease pattern: Isometric mapping of a polygon given a folding of its boundary, Proceedings of OSME6 Volume 1 Mathematics, ISBN 978-1-4704-1875-5, AMS Publishing, Washington DC, USA, (2015), 177-188.

[2] Keeling S.L. and Geretschläger R., Using Variational and PDE Methods for the Existence of Origami Models with Given Boundary Conditions, Proceedings of OSME7 Volume 2 - Mathematics, ISBN 978-1-911093-90-9, Tarquin Publishing, St. Albans, UK, (2018), 545-560.

Robert Geretschläger (robert@rgeretschlaeger.com)

BRG Keplerstraße 1, Graz, Austria

Stephen L. Keeling (stephen. keeling@uni-graz.at)

KF University of Graz, Institute for Mathematics and Scientific Computing, Heinrichstraße 36, Graz, Austria 